\title{
Light-filament dynamics and the spatiotemporal instability of the Townes profile
}

\author{
Miguel A. Porras, ${ }^{1}$ Alberto Parola, ${ }^{2}$ Daniele Faccio, ${ }^{2}$ Arnaud Couairon, ${ }^{3}$ and Paolo Di Trapani ${ }^{2,4}$ \\ ${ }^{1}$ Departamento de Física Aplicada, Universidad Politécnica de Madrid, Rios Rosas 21, ES-28003, Spain \\ ${ }^{2}$ INFM and Department of Physics, University of Insubria, Via Valleggio 11, IT-22100 Como, Italy \\ ${ }^{3}$ Centre de Physique Théorique, CNRS, École Polytechnique, F-91128, Palaiseau, France \\ ${ }^{4}$ Department of Quantum Electronics, Vilnius University, Sauletekio 9, LT 01222, Vilnius, Lithuania
}

(Received 30 October 2006; published 12 July 2007)

\begin{abstract}
The origin of the spatiotemporal filament dynamics of ultrashort pulses in nonlinear media, including axial-conical emission coupling, temporal splitting, and $\mathrm{X}$ waves, is explained by the spatiotemporal instability of spatially localized nonlinear modes. Our experiments support this interpretation.
\end{abstract}

DOI: 10.1103/PhysRevA.76.011803

PACS number(s): 42.65.Sf, 42.65.Jx

From optical pulses to Bose-Einstein condensates, from plasma instabilities to hydrodynamical or optical shocks, many nonlinear wave processes involve a dramatic increase of intensity along with compression into a strongly localized state, followed by relaxation into linear waves. In the case of ultrashort optical pulses in nonlinear self-focusing media, the dynamics generally develops in three dimensions (time and two spatial dimensions), but due to standard experimental conditions, the compression stage is dominated by a spatial self-focusing toward a strongly localized wave in space only. In the subsequent filamentary and eventual relaxation regimes an apparent stationarity hides a rich, fully spatiotemporal dynamics [1]. Temporal pulse splitting is accompanied by the emission of new temporal frequencies (axial spectral broadening), and spatial frequencies (off-axis, or conical emission), organized into X-shaped patterns in Fourier space $[2,3]$. A recent interpretation in terms of relaxation into multiple spatiotemporal $\mathrm{X}$ waves harmonizes the ultimate stationarity with some of these highly dynamic phenomena [4].

In this Rapid Communication, we aim at clarifying the mechanisms responsible for the onset of the full spatiotemporal dynamics in the filamentary regime. We build a unified interpretation based on the spatiotemporal instability of strongly localized waves in space, such as those formed upon Kerr self-focusing [5], and that points out a common origin of temporal splitting, axial and conical emissions, and $\mathrm{X}$ waves. As a minimal model, we study the temporal instability with normal dispersion of the ground state of the cubic nonlinear Schrödinger equation (NLSE) with two spatial dimensions, or Townes profile (TP) [6,7], which mimics the localized spatial profile generated at each refocusing event within an ultrashort filament [8]. Our numerical calculation of the unstable modes for all perturbation frequencies enables us to show for the first time that the spatiotemporal instability of a localized beam as the TP is featured by a couple of Y-shaped unstable modes that split each other in time, link axial and conical emission, and act as precursors of $X$ waves. A four-wave mixing-based reinterpretation of the Y-shaped instability supports its generality for spatially localized waves, and its application to interpret filament dynamics. Y-shaped unstable modes are observed at the initial stage of filamentation in our simulations and experiments.

We consider the simplest, cubic NLSE with normal time dispersion $[9,10]$ :

$$
\partial_{z} A=\frac{i}{2 k_{0}} \frac{\partial_{r}\left(r \partial_{r} A\right)}{r}-\frac{i k_{0}^{\prime \prime}}{2} \partial_{t^{\prime}}^{2} A+\frac{i \omega_{0} n_{2}}{c}|A|^{2} A,
$$

$\left(k_{0}^{\prime \prime}>0\right)$ describing the dynamics of a cylindrically symmetric wave-packet $E=A(r, t, z) \exp \left(-i \omega_{0} t+i k_{0} z\right)$ of carrier frequency $\omega_{0}$. In (1), $r$ is the radial coordinate in a plane transverse to $z, t^{\prime}=t-k_{0}^{\prime} z, k_{0}^{(n)}=d^{n} k(\omega) /\left.d \omega^{n}\right|_{\omega_{0}}$, with $k(\omega)$ the propagation constant in the medium, $n_{2}>0$ is the nonlinear refractive index, and $c$ is the speed of light in vacuum. We also consider the monochromatic, stationary, and exponentially localized solution $A=I^{1 / 2} a_{0}(\rho) \exp (i \alpha \zeta)$ to (1), where $I$ is the peak intensity, $\alpha \simeq 0.2055$, and $a_{0}(\rho)$ is the TP [see Fig. 1(a)] [7]. The scaled axial and radial coordinates are $\zeta$ $=k_{\mathrm{NL}} z$ and $\rho=\left(k_{\mathrm{NL}} k_{0}\right)^{1 / 2} r$, where $k_{\mathrm{NL}}=\omega_{0} n_{2} I / c$.

To perform the spatiotemporal instability analysis, we also introduce the dimensionless time $\tau=\left(k_{\mathrm{NL}} / k_{0}^{\prime \prime}\right)^{1 / 2} t^{\prime}$ and envelope $a=A / I^{1 / 2}$ to rewrite (1) in the form
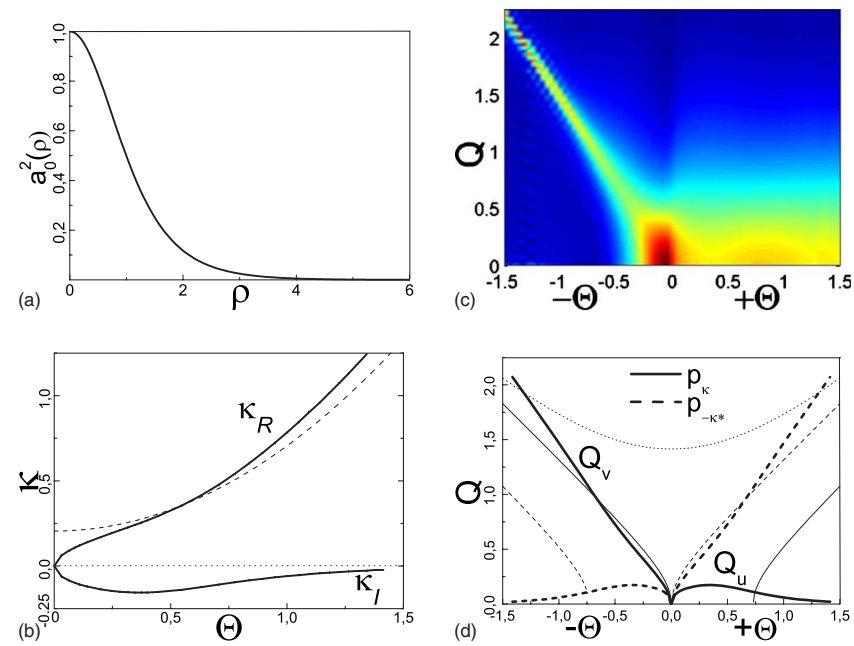

FIG. 1. (Color online) (a) Normalized TP. (b) Real and imaginary parts of the relevant complex eigenvalue. Dashed curve: $\Theta^{2} / 2+\alpha$. (c) Modulus of the spatiotemporal spectrum $\hat{p}_{\kappa}(Q, \Theta)$ of $p_{\kappa}$. The amplitudes for different $\Theta$ are arbitrarily chosen so that the energy $\int_{0}^{\infty}\left|\hat{p}_{\kappa}\right|^{2} Q d Q$ is independent of $\Theta$. (d) Thick solid and dashed curves: Characterization of the spatiotemporal spectrum of the unstable perturbations $p_{\kappa}$ and $p_{-\kappa^{\star}}$ by the dominant radial frequencies $Q_{u, v}$. Dotted curve: maximum gain curve of the plane wave. Thin solid and dashed curves: double-X spectrum. 


$$
\partial_{\zeta} a=\frac{i}{2} \frac{\partial_{\rho}\left(\rho \partial_{\rho} a\right)}{\rho}-\frac{i}{2} \partial_{\tau}^{2} a+i|a|^{2} a .
$$

Following a standard procedure [11], we consider the spatially and temporally perturbed Townes beam

$$
a=\left\{a_{0}(\rho)+\epsilon\left[u(\rho) e^{-i \Theta \tau+i \kappa \zeta}+v^{\star}(\rho) e^{i \Theta \tau-i \kappa^{\star} \zeta}\right]\right\} e^{i \alpha \zeta},
$$

where $\epsilon \ll 1$, and where $\Theta \geqslant 0$ is the absolute temporal frequency shift [in units of $\left(k_{\mathrm{NL}} / k_{0}^{\prime \prime}\right)^{1 / 2}$ ] of the perturbation with respect to the Townes frequency. Note that $u$ and $v^{\star}$ in (3) are oppositely shifted by $+\Theta$ and $-\Theta$. The perturbation (3) in the NLSE (2) leads (up to the first order in $\epsilon$ ) to

$$
\left(\begin{array}{cc}
H & a_{0}^{2} \\
-a_{0}^{2} & -H
\end{array}\right)\left(\begin{array}{l}
u \\
v
\end{array}\right)=\kappa\left(\begin{array}{l}
u \\
v
\end{array}\right)
$$

where $H=\frac{1}{2}\left[d^{2} / d \rho^{2}+(1 / \rho) d / d \rho+\Theta^{2}\right]-\alpha+2 a_{0}^{2}(\rho)$. If this eigenvalue problem for given $\Theta$ admits a solution $(u, v) \neq 0$ with eigenvalue $\kappa$ with $\kappa_{I} \equiv \operatorname{Im} \kappa<0$, then the associated perturbation (3) will grow exponentially with gain $-\kappa_{I}$, and the TP will be unstable under perturbations at frequency $\Theta$. Note also that, if $(\kappa, u, v)$ is a solution of (4) with gain $-\kappa_{I}$, then $\left(-\kappa^{\star}, v^{\star}, u^{\star}\right)$ is another solution with the same gain. These solutions represent the two growing, physically discernible perturbations

$$
\begin{gathered}
p_{\kappa}(\rho, \Theta)=\left[u(\rho) e^{-i \Theta \tau+i \kappa_{R} \zeta}+v^{\star}(\rho) e^{i \Theta \tau-i \kappa_{R} \zeta}\right] e^{-\kappa_{I} \zeta+i \alpha \zeta}, \\
p_{-\kappa^{\star}}(\rho, \Theta)=\left[v^{\star}(\rho) e^{-i \Theta \tau-i \kappa_{R} \zeta}+u(\rho) e^{i \Theta \tau+i \kappa_{R} \zeta}\right] e^{-\kappa_{I} \zeta+i \alpha \zeta},
\end{gathered}
$$

since $u$ and $v^{\star}$ are generally different and oppositely frequency shifted in $p_{\kappa}$ and $p_{-\kappa^{\star}}$. Consequently, $p_{\kappa}$ and $p_{-\kappa^{\star}}$ may grow independently, depending on how the instability is seeded.

We have solved the problem (4) numerically for each $\Theta$ $\geqslant 0$ by discretization of the differential operators on a $\rho$ grid of finite size much larger than the Townes range $d \sim 1.5$. Increasing grid points and grid size allowed us to control the accuracy of the results. For $\Theta=0$, no complex eigenvalue is found. This is in agreement with the fact that the TP does not present exponential gain under spatial perturbations, but only algebraic instability. For each $\Theta>0$, only one pair of eigenvalues $\kappa$ and $-\kappa^{\star}$ with negative imaginary part appear [see Fig. 1(b) for $\kappa$ ]. The TP is then retrieved as modulationally unstable under spatiotemporal perturbations [12], with gain $-\kappa_{I}$ limited to $\Theta \lesssim 1.5$, but without an abrupt cutoff. Unlike previous generalizations of Zakharov's results on the instability of nonlinear waveguides [11], our results are not limited to long wavelengths. The study of the unstable perturbations presented below leads, moreover, to a unified interpretation of the filament dynamics.

Figure 1(c) shows the results of the numerical calculation of the Hänkel transform $p_{\kappa}(Q, \Theta)$ of the unstable perturbation $p_{\kappa}(\rho, \Theta)$ at each frequency shift $\Theta$, where $Q$ is the radial frequency [in units of $\left(k_{\mathrm{NL}} k_{0}\right)^{1 / 2}$. The reflection of Fig. 1(c) about $\Theta=0$ yields the Hänkel transform $p_{-\kappa^{\star}}(Q, \Theta)$ of $p_{-\kappa^{\star}}(\rho, \Theta)$. These $Q-\Theta$ spectra, or spatiotemporal spectra, are particularly useful in experiments with ultrashort pulses.
Their measurement (in practice, measurement of the angularly resolved spectrum, displaying angles and wavelengths) is a powerful diagnostic from which many aspects of the spatiotemporal dynamics can be inferred [2]. For the unstable perturbation $p_{\kappa}(Q, \Theta)$ of the TP, the branch $u$ at $+\Theta$ is peaked about $Q_{u} \sim 0$ for all $+\Theta$, and hence can be identified with an axial emission at upshifted frequencies. The branch $v$ at $-\Theta$ presents instead a sharp maximum at increasing radial frequency with detuning, whose location fits well to $Q_{v}$ $\simeq \sqrt{2} \Theta$, and can be identified with a conical emission at downshifted frequencies. For $p_{-\kappa^{\star}}(Q, \Theta)$, axial emission is instead associated with downshifted frequencies and conical emission with upshifted frequencies.

These features also follow from an asymptotic analysis. Neglecting terms with $a_{0}^{2}$ in (4) and $H$ for $\rho \rightarrow \infty$, the problem (4) becomes uncoupled for $u$ and $v$, and admits an analytical solution. The bounded solutions for $\rho \rightarrow \infty$ are $u \propto H_{0}^{(1)}\left[\left(Q_{u}+i \Gamma_{u}\right) \rho\right]$ and $v \propto H_{0}^{(1)}\left[\left(Q_{v}+i \Gamma_{v}\right) \rho\right]$, where $H_{0}^{(1)}$ is the Hänkel function of first class and zero order. The real quantities $Q_{u, v}$ and $\Gamma_{u, v}$ are defined by

$$
Q_{u}+i \Gamma_{u}=\sqrt{\Theta^{2}-2 \alpha-2 \kappa}, \quad Q_{v}+i \Gamma_{v}=\sqrt{\Theta^{2}-2 \alpha+2 \kappa},
$$

with the convention of taking square roots such that $\Gamma_{u, v} \geqslant 0$. Using $H_{0}^{(1)}(s) \sim \sqrt{(2 / \pi s)} \exp [i(s-\pi / 4)]$ for large $|s|$, and ignoring constant factors and algebraical decay, $u$ and $v$ are found to be dominated at large $\rho$ by the damped oscillating behavior $\exp \left(-\Gamma_{u, v} \rho+i Q_{u, v} \rho\right)$, with radial frequencies $Q_{u, v}$. The thick curves in Fig. 1(d) represents dominant radial frequencies $Q_{u}$ and $Q_{v}$, obtained from (6), versus their respective frequency shifts, $+\Theta$ and $-\Theta$ for the perturbation $p_{\kappa}$ (solid curve) and $-\Theta$ and $+\Theta$ for the perturbation $p_{-\kappa^{\star}}$ (dashed curve). The dominant radial frequency $Q_{u}$ is close to zero, while $Q_{v}$ is well described by $Q_{v} \simeq \sqrt{2} \Theta$, as obtained above from the numerical evaluation.

Let us compare the spatiotemporal instabilities of the TP and of the plane wave solution $a=\exp (i \zeta)$ to the NLSE [9]. The problem (4) holds in the latter case if $a_{0}$ and $\alpha$ are replaced by unity. The functions $u$ and $v$ associated with the most unstable perturbations $p_{\kappa}$ and $p_{-\kappa^{\star}}$ at each frequency $\Theta$ are two plane waves with the identical transverse wave numbers $Q_{u}=Q_{v}=\sqrt{\Theta^{2}+2}$, with $\Theta$-independent gain $-\kappa_{I}=1$. The spectra $p_{\kappa}(Q, \Theta)$ and $p_{-\kappa^{\star}}(Q, \Theta)$ are then characterized by two identical hyperbolas, depicted in Fig. 1(d) as a dotted curve (see Ref. [9] for more details).

To summarize, if $\Omega$ denotes the physical (positive or negative) temporal frequency shift, and $K_{\perp}$ the physical radial frequency, the instability spectrum of the plane wave is characterized by the hyperbola $K_{\perp}=\sqrt{2 k_{0}\left(k_{\mathrm{NL}}+k_{0}^{\prime \prime} \Omega^{2} / 2\right)}$ for arbitrary $\Omega$, and the instability spectrum of the TP by the two Y-shaped curves $K_{\perp} \simeq\left(-\sqrt{2 k_{0} k_{0}^{\prime \prime} \Omega}\right.$ if $\Omega<0,0$ if $\Omega>0$ ) (and its reflection about $\Omega=0$ ), limited to $|\Omega| \lesssim 1.5 \sqrt{k_{\mathrm{NL}} / k_{0}^{\prime \prime}}$. Note the $\sqrt{2}$-fold slope of the $\mathrm{Y}$ arm in comparison with those of the hyperbola. These Y-shaped spectra point out a common origin for axial and conical emission. Typical filament spectra, displaying on-axis and X-shaped off-axis radiation, are reinterpreted here as originating from two superimposed 
Y-shaped spectra, each one linking axial to conical emission at opposite frequency bands.

The Y-shaped instability admits a simple interpretation and generalization as a four-wave-mixing interaction driven by the Kerr nonlinearity. Consider two intense, identical pump waves of frequency $\Omega=0$ propagating along the $z$ direction, and amplifying two weak, noncollinear plane waves, $u$ and $v$, at frequencies $\pm \Omega$. The axial projection of the wave vector of each wave is expressed by $k_{z}=k_{0}+k_{0}^{\prime} \Omega+k_{0}^{\prime \prime} \Omega^{2} / 2$ $-K_{\perp}^{2} / 2 k_{0}+\Delta k_{\mathrm{NL}}$ up to second order in dispersion [as in the NLSE (1)]. The last two terms account for axial wave vector shortening due to noncollinearity (for $u$ and $v$ ) and lengthening due to either self-phase (for the pump $p$ ) or cross-phase modulation (for $u$ and $v$ ). If the pumps are plane waves, the conditions of axial and transverse phase matching $\left(k_{z, u}+k_{z, v}=2 k_{z, p}\right.$ and $\left.K_{\perp, u}^{2}=K_{\perp, v}^{2} \equiv K_{\perp}^{2}\right)$, and that the crossphase-modulation wave vector shift is twice the self-phasemodulation shift [13] $\left(\Delta k_{\mathrm{NL}, u}=\Delta k_{\mathrm{NL}, v}=2 \Delta k_{\mathrm{NL}, p}=2 k_{\mathrm{NL}}\right)$, lead immediately to the hyperbolic curve $K_{\perp}$ $=\sqrt{2 k_{0}\left(k_{\mathrm{NL}}+k_{0}^{\prime \prime} \Omega^{2} / 2\right)}$.

For transversally localized pump waves, however, efficient amplification is possible with transverse mismatch such that $\left|K_{\perp, u}-K_{\perp, v}\right|<\pi / d[14]$, where $d \sim 1.5 /\left(k_{\mathrm{NL}} k_{0}\right)^{1 / 2}$ is the width of the TP. We add here the hypothesis that, owing to pump localization, plane waves collinear to the pump are preferentially amplified. We then take $u$, e.g., as collinear $\left(K_{\perp, u} \simeq 0\right)$, with $\Delta k_{\mathrm{NL}, u}=2 \Delta k_{\mathrm{NL}, p}=2 \alpha k_{\mathrm{NL}}$. Axial phase matching is then seen to require $v$ to be noncollinear $\left(K_{\perp, v} \neq 0\right)$. It is then reasonable to take $\Delta k_{\mathrm{NL}, v} \simeq 0$ for its cross-phase modulation, [4] since $v$ does not remain in the localized interaction area of the TP. With these assumptions, the sole condition of axial phase matching $\left(k_{z, u}+k_{z, v}=2 k_{z, p}\right)$ leads to the $\mathrm{Y}$ arm $K_{\perp, v} \simeq-\sqrt{2 k_{0} k_{0}^{\prime \prime}} \Omega$. Axial phase matching is then responsible for axial-conical coupling, amplification in this configuration being possible due to the transverse localization of the pump. Also, limitation of the gain bandwidth is a consequence of the maximum allowed transverse mismatch: if $K_{\perp, u} \simeq 0$, then $\left|K_{\perp, v}\right| \lesssim \pi / d$, which is satisfied for $|\Omega| \lesssim 1.5 \sqrt{k_{\mathrm{NL}} / k_{0}^{\prime \prime}}$.

The independence of the four-wave-mixing analysis on the particular localized profile stresses the generality of the Y-based time-to-space coupling mechanism, which is expected to be triggered when an intense wave remains tightly focused over a distance sufficient for a significant energy to be transferred to the phase-matched frequencies. Pulse temporal splitting is also accounted for by this mechanism. If the perturbation $p_{\kappa}$, e.g., is seeded coherently at different frequencies $\Theta$, its growth leads to the formation of a pulse. Note that $\kappa_{R}$ fits well to $\Theta^{2} / 2+\alpha$ around the values of $\Theta$ where the gain takes higher values [dashed curve in Fig. 1(b)]. The axial wave vector shift $\kappa_{R}+\alpha$ of the branch $u$ of the perturbation $p_{\kappa}$ in (5) is then approximated by $\Theta^{2} / 2+2 \alpha$, and the axial wave vector shift $-\kappa_{R}+\alpha$ of the branch $v$ by $-\Theta^{2} / 2$. The wave-mixing analysis yields the same values, which in physical units read $k_{z, u} \simeq k_{0}+k_{0}^{\prime} \Omega$ $+k_{0}^{\prime \prime} \Omega^{2} / 2+2 \alpha k_{\mathrm{NL}}$ (for $\Omega>0$ ), and $k_{z, v} \simeq k_{0}+k_{0}^{\prime} \Omega-k_{0}^{\prime \prime} \Omega^{2} / 2$ (for $\Omega<0$ ). This means that the axial part of the $\mathrm{Y}$ wave experiences the normal dispersion of the material, and the conical part the opposite anomalous dispersion as an effect
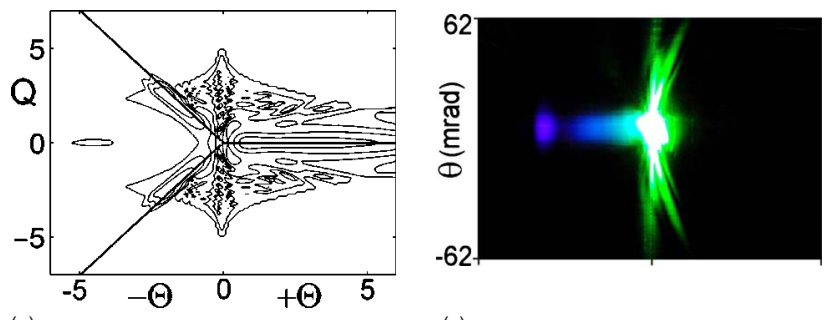

(a)

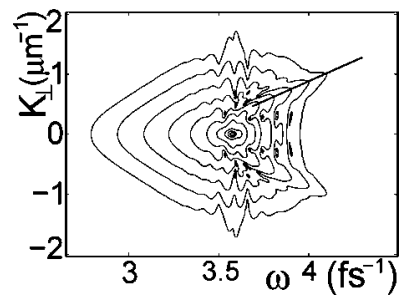

(b)

FIG. 2. (Color online) Simulated spatiotemporal spectra (in logarithmic scale, 10 decades plotted) of (a) NLSE-propagated input pulsed TP (see text), with the input spectrum subtracted; (b) filament in water at $1 \mathrm{~mm}$ beyond the collapse (see text). (c), (d) Measured angularly resolved spectra of filaments in fused silica at $E=2(\mathrm{c})$ and (d) $3 \mu \mathrm{J}$.

of its angular dispersion. Consequently, the (inverse) group velocities of the axial part, $v_{g}^{-1}=d k_{z, u} / d \Omega$ evaluated at positive $\Omega$, and of the conical part, $v_{g}^{-1}=d k_{z, v} / d \Omega$ evaluated at negative $\Omega$, are identical and equal to $k_{0}^{\prime}+k_{0}^{\prime \prime}|\Omega|$. The $\mathrm{Y}$ wave then propagates as a whole at a well-defined group velocity. For $p_{-\kappa^{\star}}$ or a reflected $\mathrm{Y}$ wave, the group velocity is instead $k_{0}^{\prime}-k_{0}^{\prime \prime}|\Omega|$, and the group velocity mismatch between the two Y waves is $2 k_{0}^{\prime \prime}|\Omega|$. At the stage of well-developed instability, the spectrum will exhibit sidebands at the maximum gain frequencies $|\Omega| \simeq 0.4\left(k_{\mathrm{NL}} / k_{0}^{\prime \prime}\right)^{1 / 2}$, and hence the group velocity mismatch approaches $0.8\left(k_{\mathrm{NL}} k_{0}^{\prime \prime}\right)^{1 / 2}=0.8\left(\omega_{0} k_{0}^{\prime \prime} n_{2} I / c\right)^{1 / 2}$, in agreement with the dependence on pump intensity and material properties in filamentation experiments [2].

It follows from our analysis that the onset of downshifted axial emission must be accompanied by upshifted conical emission, and vice versa, and that these two events may occur independently. Our simulations and experiments support this interpretation. First, we perturbed asymmetrically a TP by making it pulsed (i.e., nonmonochromatic) with unequal durations in its leading and trailing parts (dimensionless durations $\Delta \tau=0.87$ and 29 ). This perturbation, however, does not break the initial symmetry about $\Theta=0$ of the spectral intensity in the $Q-\Theta$ plane. Propagation under the NLSE (1) accounts for dispersion and nonuniform spatial dynamics at different slices of the pulse, and results in an asymmetric spectrum, which, subtracted from the input one (to visualize the newly generated frequencies), yields the Y-shaped spectrum of Fig. 2(a) (at $\zeta=7.6$ ), with the off-axis tail fitting to the expected slope $\sqrt{2}$ (thick line).

Y-shaped spectra are also formed at the earlier stage of filamentation. We simulated in real-world variables the filamentation in water of an input $(z=0 \mathrm{~cm})$ Gaussian pulse of $200 \mathrm{fs}$ duration (full width at half maximum), $76 \mu \mathrm{m}$ Gaussian width, and energy $2 \mu \mathrm{J}$ (peak intensity $I_{0}$ 
$\simeq 10^{11} \mathrm{~W} / \mathrm{cm}^{2}$ ) at $527 \mathrm{~nm}$ carrier wave length. The material parameters and propagation equation, which includes Kerr nonlinearity, diffraction, dispersion at any order, nonlinear losses, and plasma defocusing, are thoroughly explained in Ref. [15], where X-shaped spectra well beyond collapse were investigated. Collapse is regularized by non-Kerr effects from $z=1.1$ to $1.2 \mathrm{~mm}$, where strong localization along with nearly constant peak intensity $I \simeq 7 \times 10^{12} \mathrm{~W} / \mathrm{cm}^{2}$ is observed. At $1.2 \mathrm{~cm}$ [Fig. 2(b)] the spectrum displays half-axial and half-conical emission with the slope $\sqrt{2 k_{0} k_{0}^{\prime \prime}}$ $\simeq 1.33 \mathrm{fs} / \mu \mathrm{m}$ (thick line), while no particular spectral structure was observed at $1.1 \mathrm{~cm}$. The growth of the Y-shaped spectrum in about $1 \mathrm{~mm}$ is consistent with a characteristic gain length $\left(k_{\mathrm{NL}}\left|\kappa_{I}\right|\right)^{-1} \simeq 0.5 \mathrm{~mm}$ estimated from the peak intensity $I$ in this region.

In the experiments, we used a 15-cm-long fused silica sample as Kerr medium. 200-fs-long pulses at $527 \mathrm{~nm}$ delivered from a $10 \mathrm{~Hz} \mathrm{Nd:glass} \mathrm{mode-locked} \mathrm{and} \mathrm{regeneratively}$ amplified laser (Twinkle, Light Conversion) were spatially filtered and focused with a lens of $50 \mathrm{~cm}$ focal length. The pulses then entered into the sample, whose input facet was placed at $52 \mathrm{~cm}$ from the lens, and formed a single filament for input energies $E \gtrsim 2 \mu \mathrm{J}$. Single-shot, angularly resolved spectra of the filament at the output facet were measured with an imaging spectrometer and a charge-coupled device camera, as described in detail in [2]. At $2 \mu \mathrm{J}$ in fused silica, the filament is formed just before the output facet of the sample, the Y-shaped (blue axial, red conical) spectrum of Fig. 2(c) then being observed. At $3 \mu \mathrm{J}$, the filament is formed closer to the input facet. The double-Y spectrum of Fig. 2(d) then corresponds to a longer filament path within the sample. The faster growth of one of the two unstable perturbations supposes some unbalancing in their seeds, arising, as expected, from the higher-order effects that arrest collapse.

Extending our analysis, we may venture an explanation of the fact that two $X$ waves are commonly observed in filaments at later stages of propagation $[3,4]$. Consider, within the four-wave-mixing approach, the possible effects on the instability spectrum of the strong temporal localization of the pump, as may take place upon (possibly multiple) splitting. For a spatially and temporally localized pump of central frequency $\Omega=0$, axial wave vector $k_{z, p}=k_{0}+\Delta k_{\mathrm{NL}}$, and propagating at a group velocity $v_{g}$ different from that of a plane pulse at $\Omega=0$ (as for a Y wave), new plane waves $u$ and $v$ at opposite frequencies $\pm \Omega$ are expected to be preferentially amplified if in addition to axial phase matching $\left(k_{z, u}+k_{z, v}\right.$ $=2 k_{p}$ ), the velocity of the group formed by $u$ and $v$ matches the velocity of the pump [i.e., the inverse beating group velocity verifies $\left.\left(k_{z, u}-k_{z, v}\right) / 2|\Omega|=1 / v_{g}\right]$. These two conditions yield the linear relation $k_{z}=k_{0}+\Delta k_{\mathrm{NL}}+\Omega / v_{g}$ for $u$ (at $\Omega>0$ ) and for $v($ at $\Omega<0)$. Since the transversal and axial projections of the wave vector are related by $K_{\perp}=\sqrt{2 k_{0}\left[k(\Omega)-k_{z}\right]}$ [in the paraxial approximation involved in the NLSE (1)], where $k(\Omega)=k_{0}+k_{0}^{\prime} \Omega+k_{0}^{\prime \prime} \Omega^{2} / 2$, we obtain $K_{\perp}$ $=\sqrt{2 k_{0}\left(\beta+\gamma \Omega+k_{0}^{\prime \prime} \Omega^{2} / 2\right)}$, which is the dispersion curve of a frequency-gap $\mathrm{X}$-wave mode $[4,16]$ with $\beta=-\Delta k_{\mathrm{NL}}$ and $\gamma$ $=k_{0}^{\prime}-1 / v_{g}$. If this process takes place for the two split-off pulses with opposite group velocities, the two $\mathrm{X}$ waves of Fig. 1(d), the thin solid and dashed curves, are formed. On nonlinearity relaxation $\left(\Delta k_{\mathrm{NL}} \rightarrow 0\right)$ at larger $z$, one branch of each $\mathrm{X}$ wave is seen to pass through $\left(K_{\perp}, \Omega\right)=0$, as observed $[3,4]$.

In conclusion, $\mathrm{Y}$ waves, emerging from the spatiotemporal instability of spatially localized modes in Kerr dynamics, constitute the missing link between axial and conical emission, and allow us to interpret temporal splitting and $\mathrm{X}$-wave formation. These results provide a unified view of ultrashort pulse filamentation, and can find application to all nonlinear waves involving subdimensional localization, such as $\chi^{(2)}$ solitons, matter waves with anisotropic confinement, plasma waves [17], etc.

M.A.P. acknowledges Project No. HI2004-078. P.D.T. acknowledges Marie Curie Chair project STELLA, Grant No. MEXC-CT-2005-025710.
[1] A. Couairon and A. Mysyrowicz, Phys. Rep. 441, 47 (2006).

[2] D. Faccio et al., J. Opt. Soc. Am. B 22, 862 (2005).

[3] M. Kolesik, E. M. Wright, and J. V. Moloney, Phys. Rev. Lett. 92, 253901 (2004).

[4] D. Faccio et al., Phys. Rev. Lett. 96, 193901 (2006).

[5] K. D. Moll, A. L. Gaeta, and G. Fibich, Phys. Rev. Lett. 90, 203902 (2003).

[6] G. Fibich and G. Papanicolaou, SIAM J. Appl. Math. 60, 183 (1999).

[7] R. Y. Chiao, E. Garmire, and C. H. Townes, Phys. Rev. Lett. 13, 479 (1964)

[8] M. Mlejnek, M. Kolesik, E. M. Wright, and J. V. Moloney, Math. Comput. Simul. 56, 563 (2001).

[9] L. W. Liou, X. D. Cao, C. J. McKinstrie, and G. P. Agrawal, Phys. Rev. A 46, 4202 (1992).
[10] G. G. Luther, A. C. Newell, J. V. Moloney, and E. M. Wright, Opt. Lett. 19, 789 (1994).

[11] V. E. Zakharov and A. M. Rubenchik, Sov. Phys. JETP 38, 494 (1974).

[12] Y. R. Shen, The Principles of Nonlinear Optics (WileyInterscience, New York, 1984).

[13] R. Alfano and S. Shapiro, Phys. Rev. Lett. 24, 584 (1970).

[14] A. Penzkofer and H. J. Lehmeier, Opt. Quantum Electron. 25, 815 (1993).

[15] A. Couairon, E. Gaizauskas, D. Faccio, A. Dubietis, and P. Di Trapani, Phys. Rev. E 73, 016608 (2006).

[16] M. A. Porras and P. Di Trapani, Phys. Rev. E 69, 066606 (2004).

[17] N. E. Andreev, L. M. Gorbunov, P. Mora, and R. R. Ramazashvili (unpublished). 